\title{
Mimicry of an acute pseudocyst by a gastrointestinal duplication cyst in a 14-year-old boy
}

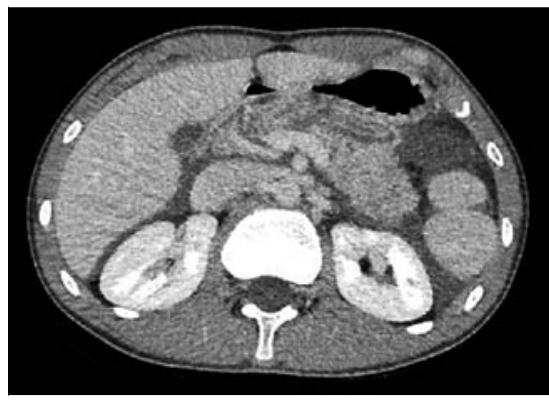

- Fig. 1 Computed tomography scan of the abdomen showing a demarcated peripancreatic fluid collection anterior to the pancreas, with free surrounding intra-abdominal fluid.

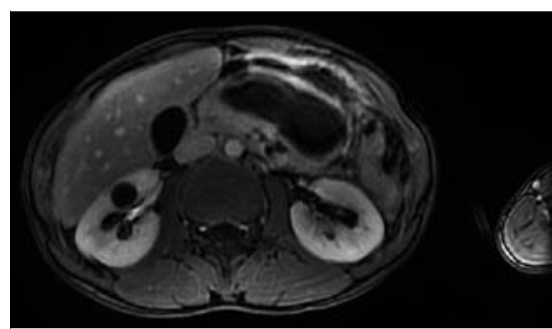

- Fig. 2 Magnetic resonance imaging scan of the abdomen 6 days after initial presentation showing a re-established tense bilobular encapsulated large fluid collection.

A 14-year-old boy was urgently admitted to the hospital because of acute abdominal pain, vomiting, abdominal tenderness, and elevated lipase levels of up to 2000 U/L. An initial basic work-up did not show anything to suggest a conventional etiology. The diagnosis of pancreatitis was put forward, which was confirmed by an abdominal computed tomography (CT) scan. Remarkably, the scan additionally revealed a demarcated flaccid peripancreatic fluid collection anterior to the pancreas, with free surrounding intra-abdominal fluid (\$Fig. 1). At that time, it was assumed that a pre-existing acute pseudocyst had ruptured spontaneously

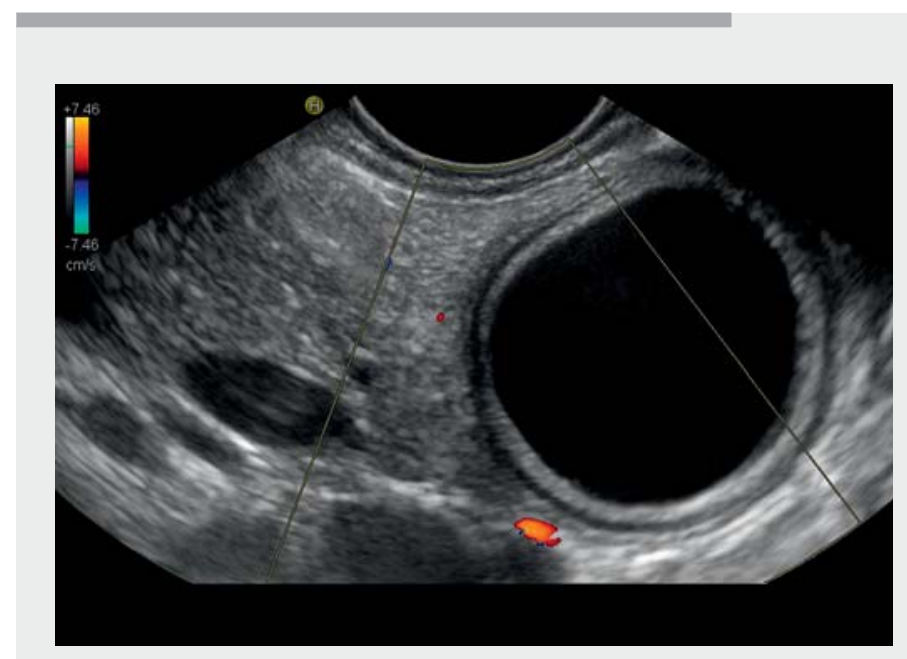

$\nabla$ Video 1 Endoscopic ultrasound evaluation of a suspected symptomatic pancreatic pseudocyst in a 14-year-old boy with episodes of pancreatitis.

and was causative of the clinical presentation. A further work-up by means of magnetic resonance imaging (MRI) 6 days after admission, and 3 days after the previously mentioned CT scan, strangely enough exhibited a fully re-established tense bilobular encapsulated fluid collection (length $8.3 \mathrm{~cm}$, width $4.1 \mathrm{~cm}$ ) ( Fig.2), instead of the previously described flaccid collection. Recurrence of the initial acute pseudocyst was suspected.

Given the symptomatic and recurrent nature of the pseudocyst, the patient was referred for endoscopic drainage. At endoscopic ultrasound (EUS) before the drainage, the presumed peripancreatic fluid collection appeared as a well-demarcated cystic lesion, showing sonolucent content and the absence of vascular signal ( Video 1). Upon closer inspection, its wall displayed the typical fivelayered architecture of the gastrointestinal wall and, furthermore, showed clear peristaltic contractions.
The diagnosis of a "pseudocyst-mimicking” gastrointestinal duplication cyst (GDC) was therefore put forward. The patient was referred for surgical exploration, which confirmed our premise. A latero-lateral cystogastrostomy was performed. A full-thickness biopsy authenticated the typical intestinal layered architecture and the presence of intestinal-type epithelium. The patient had an uneventful course after surgery.

The possibility of GDCs should be kept in mind in children presenting with cystic lesions in the upper abdomen. Distinguishing these from pancreatic pseudocysts may prove a real diagnostic dilemma, which can be overcome by a detailed morphological study using EUS.

Endoscopy_UCTN_Code_CCL_1AZ_2AH

\section{Competing interests}

The authors declare that they have no conflict of interest. 
The authors

Thomas Billiet ${ }^{1,2}$, Ann Elewaut ${ }^{2}$, François d'Heygere ${ }^{2}$, Gert de Hertogh ${ }^{3}$, Raymond Aerts $^{4}$, Chris Verslype ${ }^{1,2}$, Wim Laleman ${ }^{1,5}$ (-)

1 Department of Gastroenterology and Hepatology, Section of Liver and Biliopancreatic disorders, University Hospitals Leuven, KU Leuven, Leuven, Belgium

2 Department of Gastroenterology and Hepatology, AZ Groeninge, Kortrijk, Belgium

3 Department of Pathology, University Hospitals Leuven, KU Leuven, Leuven, Belgium

4 Department of Abdominal Surgery, Section of Hepatobiliary and Pancreatic Surgery, University Hospitals Leuven, KU Leuven, Leuven, Belgium

5 Department of Chronic Diseases, Metabolism and Ageing (CHROMETA), KU Leuven, Leuven, Belgium
Corresponding author

\section{Wim Laleman, MD, PhD}

Department of Gastroenterology \&

Hepatology, Section of Liver and

Biliopancreatic disorders, University

Hospitals Leuven, KU Leuven, Herestraat 49,

Leuven 3000, Belgium

wim.laleman@uzleuven.be

Bibliography

Endoscopy 2022; 54: E271-E272

DOI 10.1055/a-1508-5546

ISSN 0013-726X

published online 18.6.2021

(c) 2021. Thieme. All rights reserved.

Georg Thieme Verlag KG, Rüdigerstraße 14

70469 Stuttgart, Germany
ENDOSCOPY E-VIDEOS

https://eref.thieme.de/e-videos

Endoscopy E-Videos is an

ry open access online section,

回侜: reporting on interesting cases

and new techniques in gastroenterological endoscopy. All papers include a high quality video and all contributions are freely accessible online. Processing charges apply (currently EUR 375), discounts and wavers acc. to HINARI are available.

This section has its own submission website at

https://mc.manuscriptcentral.com/e-videos 